Article

\title{
Application of an Adaptive Multi-Population Parallel Genetic Algorithm with Constraints in Electromagnetic Tomography with Incomplete Projections
}

\author{
Changfang Guo, Zhen Yang *, Xiang Wu, Tingjiang Tan and Ke Zhao \\ Key Laboratory of Deep Coal Resource Mining, Ministry of Education of China, School of Mines, \\ China University of Mining \& Technology, Xuzhou 221116, China \\ * Correspondence: yangzhen@cumt.edu.cn; Tel.: +86-186-5179-8874
}

Received: 11 June 2019; Accepted: 25 June 2019; Published: 27 June 2019

check for updates

\begin{abstract}
Electromagnetic tomography technology (EMT) is widely used in underground energy exploration. Limited by objective conditions, the collected projection data of electromagnetic waves are sparse and incomplete. Therefore, a study of the tomographic inversion algorithm of EMT based on incomplete projection data has an important guiding significance for the exploitation of underground energy. As a global optimization probability search algorithm, the simple genetic algorithm (SGA) has been widely used in the process of tomographic inversion. However, SGA evolves through a single population, and the values of crossover and mutation probability are always fixed, so there are risks of premature convergence and poor local search ability. To improve the performance of the SGA, a new approach of adaptive multi-population parallel genetic algorithm (AMPGA) with constraints is proposed in this paper. First, the AMPGA makes full use of multi-group adaptive co-evolution to improve the local and global search ability of SGA and restrain the risk of premature convergence. Then, the introduction of prior information as a constraint makes the results clearer and more accurate. The proposed algorithm has been verified in a numerical experiment and field tests, and the results show that the proposed algorithm can well balance global and local search capabilities, which offers a more realistic and stable tomographic result.
\end{abstract}

Keywords: EMT; underground energy exploration; incomplete projection; SGA; AMPGA; constraint

\section{Introduction}

With continuous energy shortages, accurate exploration of underground energy has become the mainstream of international research [1]. Electromagnetic tomography (EMT) has particular advantages over other exploration methods, such as drilling methods, 3-D seismic exploration, and channel wave tomography, as it can realize high imaging resolution, has convenient construction, and is non-hazardous [2-5]. EMT has been widely used in the exploration of coal, oil, natural gas, and other mineral resources [6-8]. However, in the process of exploration, the ray coverage angle in the observation system is limited by field detection conditions, and the electromagnetic wave projection data obtained are always incomplete. Therefore, the most challenging task of EMT is solving for a large, sparse, and ill-conditioned tomographic inversion model.

In the current study, various random global search iterative methods that have attracted greater attention because of their decreased dependence on the initial model have been introduced to deal with the above problems. The simple genetic algorithm (SGA) is a highly parallel, random, and adaptive global optimization probability search algorithm developed from natural selection and evolutionary mechanisms in the biological world [9]. Since it does not depend on gradients in optimization, it has 
strong robustness and global search ability. Therefore, SGA is widely used in machine learning, pattern recognition, mathematical programming, and other fields [10-15]. At the same time, SGA has also been employed as the solution of geophysical tomographic inversion problems [16-19]. However, many shortcomings have also been exposed by the wide application of SGA and deeper research. In the evolution of SGA, the choice of crossover probability and mutation probability often determines the balance of global and local search ability of the algorithm. The values of crossover and mutation probability in SGA are always fixed, so there are risks of premature convergence and poor local searchability $[20,21]$.

To make full use of the global evolutionary characteristics of SGA and restrain its shortcomings of premature convergence and poor local search ability in the inversion process of EMT, an adaptive multi-population parallel genetic algorithm (AMPGA) with constraints is proposed in this paper. On the basis of SGA, global and local search capabilities are improved by multi-group parallel evolution with different genetic parameters. Then, the crossover and mutation probabilities are adaptively adjusted according to the fitness value of each individual in each population, which further improves the local search ability and reduces the possibility of premature convergence. Finally, the tomographic resolution is further improved by adding the constraint with prior information.

In this research, the proposed algorithm of AMPGA with constraints has been developed combined with a numerical experiment and field test to find a better solution for the tomographic inversion model with incomplete projections. The root mean square error (RMSE) between the computed and the picked attenuation coefficient is set as the fitness reference in the optimized calculation. By repeating experiments on the algorithms, the variation characteristics of the RMSE curve and the average value of the RMSE are used to verify the accuracy and stability of the algorithms. The results show that the accuracy and stability of image reconstruction of the proposed algorithm in the EMT is significantly improved compared with the SGA, and it has important guiding significance for accurate energy exploration and mining.

\section{Proposed Method}

\subsection{Inversion Model}

In this paper, the electromagnetic wave tomography inversion model is established for detecting the distribution of abnormal areas within the working face in a coal mine. Due to the different electrical properties (resistivity and dielectric constant) of different media, their absorptivity to electromagnetic waves is also different. Coal with high resistivity has weak absorption of electromagnetic waves, which is beneficial to its penetration. The anomalous structures with lower resistivity, such as faults, collapse columns, and voids, have stronger shielding effects on electromagnetic wave absorption, resulting in significant energy attenuation [22-24]. Therefore, the distribution of abnormal structures within the working face can be predicted by analyzing the variation of electromagnetic field intensity in the working face.

The survey of EMT is carried out between two tunnels of the working face; the electromagnetic wave with a certain frequency is emitted in one roadway and received in another roadway. The fixed points method is always adopted for observation, which is fast, efficient, and easy to coordinate. The transmitter is relatively fixed in a certain period of time, and the receiver observes its field strength point by point in a certain range, which is shown in Figure 1. 


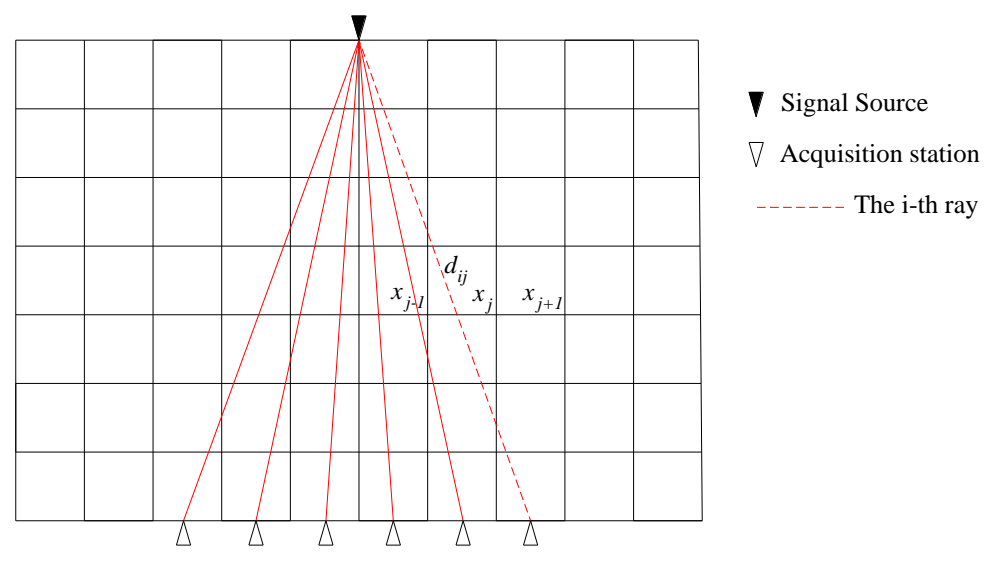

Figure 1. Fixed point observation sketch.

The $H_{i}$ received by the acquisition station of the $i$-th ray is

$$
H_{i}=H_{0}-\beta r-\ln r
$$

where $H_{0}$ is the original field strength emitted at the signal source, $\beta$ is the attenuation coefficient, and $r$ is the length of the $i$-th ray.

After the detection area is discretized as shown in Figure $1, x_{j}$ is the attenuation coefficient of the $j$-th grid, and $d_{i j}$ is the interception of the $i$-th ray passing through the $j$-th grid. Therefore, in the path of $i$-th ray, the expression of $\beta r$ can also be described as

$$
\beta r=\sum_{j=1}^{n} d_{i j} x_{j} .
$$

If $y_{i}$ is defined as

$$
y_{i}=H_{0}-H_{i}-\ln r
$$

then

$$
y_{i}=\sum_{j=1}^{n} d_{i j} x_{j}
$$

Assuming that the total number of rays is $A$ and the total number of grids is $B$, the matrix equation can be obtained from multiple observations with different signal sources:

$$
\left[\begin{array}{cccc}
d_{11} & d_{12} & \ldots & d_{1 B} \\
d_{21} & d_{22} & \ldots & d_{2 B} \\
\vdots & \vdots & \vdots & \vdots \\
d_{A 1} & d_{A 2} & \ldots & d_{A B}
\end{array}\right]\left[\begin{array}{c}
x_{1} \\
x_{2} \\
\vdots \\
x_{B}
\end{array}\right]=\left[\begin{array}{c}
y_{1} \\
y_{2} \\
\vdots \\
y_{A}
\end{array}\right] .
$$

It can also be expressed as

$$
[\mathrm{D}][\mathrm{X}]=[\mathrm{Y}]
$$

where $[\mathrm{D}]$ is the $\left(A^{*} B\right)$ matrix operator, which is the intercept of each ray in all meshes; [Y] is the A dimensional constant vector acquired from the EMT survey, which is related to the measured field strength; $[X]$ is the $B$ dimensional attenuation coefficient vector, which is the goal of tomographic inversion.

Limited by field construction conditions, the number of rays is always far less than the number of grids. Therefore, an approximate solution of a sparse, ill-conditioned Equation (6) must be found. In this paper, the value of $[\mathrm{X}]$ is required when the objective function $(F)$ reaches the global minimum 
according to the AMPGA. $F$ is defined as the root mean square error (RMSE) of the value of the attenuation coefficient between that computed and that acquired from the survey:

$$
F=\sqrt{\frac{1}{A} \sum_{i=1}^{A}\left(y_{i}-\sum_{j=1}^{B} d_{i j} x_{j}\right)^{2}}
$$

On this basis, this paper attempts to improve the effect of tomographic inversion by adding the prior information to constrain the iteration direction. The prior information is acquired from the tunnels, and the mathematical model that needs to be reconstructed after introducing a prior constraint is described by

$$
\left\{\begin{array}{l}
\min F \\
\text { s.t } \quad x_{j} \geq 0 \\
\text { s.t } \quad x_{j}= \begin{cases}\frac{1}{B} \sum_{j=1}^{B} x_{j} & j \in Q \\
x_{j} & j \notin Q\end{cases}
\end{array}\right.
$$

where $Q$ is the set of grid numbers corresponding to the revealed prior information.

\subsection{The AMPGA Algorithm with Constraint}

The goal of this paper is to search for the attenuation coefficient closer to the globally optimal solution corresponding to the mathematical model (8). The algorithm of AMPGA with constraints is employed to cover the shortage of SGA, such as premature convergence and poor local search ability, and the specific process is shown in Figure 2.

(1) Initialize the involved parameters.

(a) $A$ is total number of rays, and $B$ is the total number of grids.

(b) Set $g=0$, where $g$ is the current number of generations.

(c) Set $M P=10$ as the number of the populations. The algorithm breaks through the framework of SGA's genetic evolution by a single population and introduces multiple populations simultaneously to optimize the search, which improves the global and local search ability.

(d) Set NIND $=40$ as the size of each population.

(e) Set $G G A P=0.95$ as the generation gap.

(f) Set $M A X G E N=500$ as the maximum number of generations.

(g) Set $P o p \in(0,+\infty)$, the corresponding absorption coefficient of each individual in the population is greater than 0 .

(h) Determination of the crossover probability $P_{c}(g, p, n)$ and the mutation probability $P_{m}(g, p$, $n)$, where $P_{c}(g, p, n)$ is the crossover probability of the $n$-th individual in the $p$-th population when it evolved to the $g$-th generation, $P_{m}(g, p, n)$ is the mutation probability of the $n$-th individual in the $p$-th population when it evolved to the $g$-th generation.

The values of the crossover probability $P_{c}$ and the mutation probability $P_{m}$ determine the equilibrium of the algorithm's global search and local search capabilities. Many scholars recommend choosing a larger $P_{c}$ and a smaller $P_{m}$ [25]; however, there are still countless ways to value $P_{c}$ and $P_{m}$. For different choices, the optimization results vary greatly. In AMPGA, different genetic parameters are set for different populations, which improves the global and local search ability [26-28]. In this paper, the range of $P_{c}$ is defined between $\left(P_{c}\right)_{\min }$ and $\left(P_{c}\right)_{\max }$, and the range of $P_{m}$ is defined between $\left(P_{m}\right)_{\min }$ and $\left(P_{m}\right)_{\max }$.

$$
\left\{\begin{array}{l}
\left(P_{c}\right)_{\text {max }}=0.9,\left(P_{c}\right)_{\text {min }}=0.5 \\
\left(P_{m}\right)_{\text {max }}=0.05,\left(P_{m}\right)_{\text {min }}=0.001
\end{array}\right.
$$




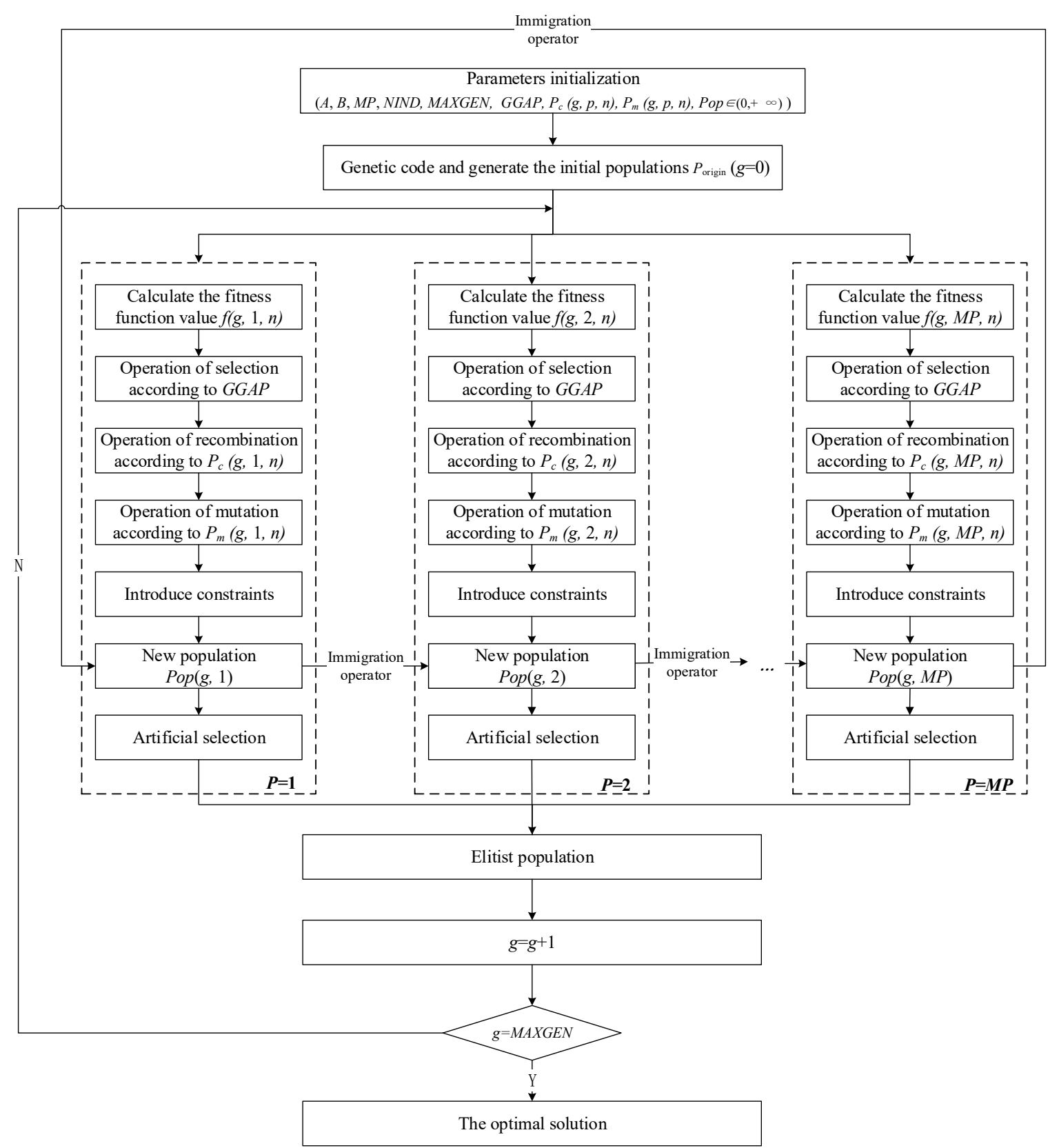

Figure 2. Flow chart of adaptive multi-population parallel genetic algorithm (AMPGA) with constraints.

In order to improve the diversity of the population and global search ability, multiple populations are employed to evolve in parallel [29-31]. Before evolution $\left(g_{0}=0\right)$, the initial genetic parameters are defined respectively:

$$
\left\{\begin{array}{c}
P_{c 1}\left(g_{0}, p\right)=\left(P_{c}\right)_{\text {min }}+\left(\left(P_{c}\right)_{\text {max }}-\left(P_{c}\right)_{\text {min }}\right) * \delta \\
P_{m 1}\left(g_{0}, p\right)=\left(P_{m}\right)_{\text {min }}+\left(\left(P_{m}\right)_{\text {max }}-\left(P_{m}\right)_{\text {min }}\right) * \delta
\end{array}\right.
$$

where $\delta$ is an number randomly generated between 0 and $1, P_{c 1}\left(g_{0}, p\right)$ is the $p$-th population's initial crossover probability, $P_{m 1}\left(g_{0}, p\right)$ is the $p$-th population's initial mutation probability.

On the other hand, in order to further improve the local search ability of the model, the idea of the adaptive genetic algorithm [32,33] is introduced. In the course of evolution, the probability of crossover and mutation of each individual in each population is adjusted. The individuals with high fitness are given lower probability of crossover and mutation, which enables the solution to be protected into 
the next generation. The individuals with low fitness are given higher probability of crossover and mutation, which enables the solution to be eliminated quickly. Therefore, the adaptive crossover and mutation probability can be used to improve the local convergence speed and search ability.

The lower limit of adaptive change of the crossover and mutation probability for each individual in each population is set as

$$
\left\{\begin{array}{l}
P_{c 2}\left(g_{0}, p, n\right)=P_{c 1}\left(g_{0}, p, n\right)-0.3 \\
P_{m 2}\left(g_{0}, p, n\right)=P_{m 1}\left(g_{0}, p, n\right) / 100
\end{array} .\right.
$$

Therefore, in the evolution process through AMPGA, the crossover and mutation probability of each individual is defined as

$$
\begin{aligned}
& P_{c}(g, p, n)= \begin{cases}P_{c 1}\left(g_{0}, p, n\right)-\frac{\left(P_{c 1}\left(g_{0}, p, n\right)-P_{c 2}\left(g_{0}, p, n\right)\right) *\left(f_{\max }(g, p)-f_{b}(g, p, n)\right)}{f_{\max }(g, p)-f_{\text {avg }}(g, p)} & f_{b}(g, p, n) \geq f_{\text {avg }}(g, p) \\
P_{c 1}\left(g_{0}, p, n\right) & f_{b}(g, p, n)<f_{\text {avg }}(g, p)\end{cases} \\
& P_{m}(g, p, n)= \begin{cases}P_{m 1}\left(g_{0}, p, n\right)-\frac{\left(P_{m 1}\left(g_{0}, p, n\right)-P_{m 2}\left(g_{0}, p, n\right)\right) *\left(f_{\max }(g, p)-f(g, p, n)\right)}{f_{\max }(g, p)-f_{\text {avg }}(g, p)} & f(g, p, n) \geq f_{\text {avg }}(g, p) \\
P_{m 1}\left(g_{0}, p, n\right) & f(g, p, n)<f_{\text {avg }}(g, p)\end{cases}
\end{aligned}
$$

where $f_{b}(g, p, n)$ represents the fitness value of the larger value between the two individuals to be crossed in the $g$-th generation of the $p$-th population, $f(g, p, n)$ is the $n$-th individual's fitness value in the $g$-th generation of the $p$-th population, $f_{a v g}(g, p)$ is the average fitness value in the $g$-th generation of the $p$-th population, $f_{\max }(g, p)$ is the largest fitness value in the $g$-th generation of the $p$-th population.

(2) Evolutionary operations of AMPGA.

(a) According to the genetic parameter settings, initial populations $P_{\text {origin }}(g=0)$ are randomly generated within a certain prior constraint range (the value of each individual in the population is greater than 0$)$.

$$
P_{\text {origin }}=\{\operatorname{Pop}(g, 1), \operatorname{Pop}(g, 2), \ldots, \operatorname{Pop}(g, p), \ldots, \operatorname{Pop}(g, M P)\}
$$

where the Pop $(g, p)$ is the $p$-th population in the $g$-th generation.

$$
\begin{gathered}
P o p(g, p)=\{P(g, p, 1), P(g, p, 2), \ldots, P(g, p, n), \ldots, P(g, p, N I N D)\} \\
P(g, p, n)=\left[\begin{array}{c}
x_{1}(g, p, n) \\
x_{2}(g, p, n) \\
\vdots \\
x_{j}(g, p, n) \\
\vdots \\
x_{N}(g, p, n)
\end{array}\right]
\end{gathered}
$$

where $P(g, p, n)$ is the attenuation coefficient model of the tomographic inversion region represented by the $n$-th individual in the $g$-th generation of the $p$-th population. Among them, $p=1,2,3 \ldots M P ; j=$ $1,2,3 \ldots B ; n=1,2,3 \ldots$ NIND.

Binary coding is carried out for each individual in all populations.

(b) Calculate the objective function value $F(g, p, n)$ of each individual in each population, and obtain the corresponding fitness value $f(g, p, n)$ according to the linear ordering method with a pressure difference $(S P=2)$ :

$$
f(g, p, n)=2-S P+\frac{2(S P-1)(\text { Pos }-1)}{M P-1}
$$

where Pos is the order according to the objective function value $F(g, p, n)$. 
(c) Selection operation. The purpose of selection is to select good individuals from the current population, so that they can have the opportunity to reproduce their offspring as fathers. According to the fitness value of each individual and the generation gap GGAP, some excellent individuals are selected from the previous generation and inherited to the next generation. Among them, individuals with high fitness are more likely to be selected to contribute to the next generation with more than one offspring, which also reflects Darwin's principle of survival of the fittest.

(d) Crossover operation. A new generation of individuals is produced by crossover operation. Individuals within each population are selected randomly to exchange part of their chromosomes according to $P_{c}(g, p, n)$.

(e) Mutation operation. For each individual in each population, the gene value at one or some loci is changed to other alleles according to $P_{m}(g, p, n)$. Mutation operation not only maintains the population diversity but also ensures the local search ability. Like the biological world, the probability of mutations occurring in genetic algorithms is low. Mutation operations provide opportunities for the creation of new individuals.

(f) Introducing constraints. Adding the prior information can help improve the accuracy of the inversion image. The results from the mutation operations are corrected according to the disclosed geological information, and the correction formula is described as follows:

$$
x_{j}(g, p, n)=\left\{\begin{array}{l}
\frac{1}{B} \sum_{j=1}^{B} x_{j}(g, p, n) \quad j \in Q \\
x_{j}(g, p, n) \quad j \notin Q
\end{array} .\right.
$$

(g) Immigration operation. Different populations are relatively independent and are linked by immigration operators. The immigration operator introduces the optimal individuals of various populations in the evolution process periodically (every other generation in this paper) into other populations and realizes the information exchange between the populations.

The specific operation rule is to replace the worst individual in the target population with the best individual in the source population. That is:

$$
P\left(g, p+1, n_{\min }\right)=P\left(g, p, n_{\max }\right)
$$

where $P\left(g, p+1, n_{\min }\right)$ is the worst individual in the target population, and $P\left(g, p, n_{\max }\right)$ is the best individual in the source population.

(h) Artificial selection operation. After the end of each generation, the optimal individuals of each population are selected by artificial operators to store them in elite populations. Elite populations do not have genetic operations such as selection, crossover, and mutation, to ensure that the optimal individuals produced by different populations during evolution are not destroyed or lost.

(i) Select the best individual from the elite population and Iter $=$ Iter +1 .

(j) If Iter = MAXGEN, the process of the evolution will stop, and the result of the attenuation coefficient model is acquired. Otherwise, go to (b).

\section{Numerical Experiment and Analysis}

To demonstrate the effectiveness of the presented algorithm, we implemented it on a PC ( $3.3 \mathrm{GHz}$ CPU, 8.0 G memory), and all algorithms were implemented in the MATLAB 2017b programming environment (MathWorks Inc., Natick, MA, USA, 2017). The numerical experimental results using the algorithm of AMPGA with constraints are illustrated in this section. According to the characteristics of the narrow working face of the coal mine, the scale of the model was $600 \times 200 \mathrm{~m}$ in this numerical simulation test. As shown in Figure 3, assume that there are two abnormal structures with different shapes in the working face. The attenuation coefficient of the abnormal structures was $\beta_{1}=1 \mathrm{~dB} / \mathrm{m}$, and the attenuation coefficient of the background area was $\beta_{2}=0.5 \mathrm{~dB} / \mathrm{m}$. 


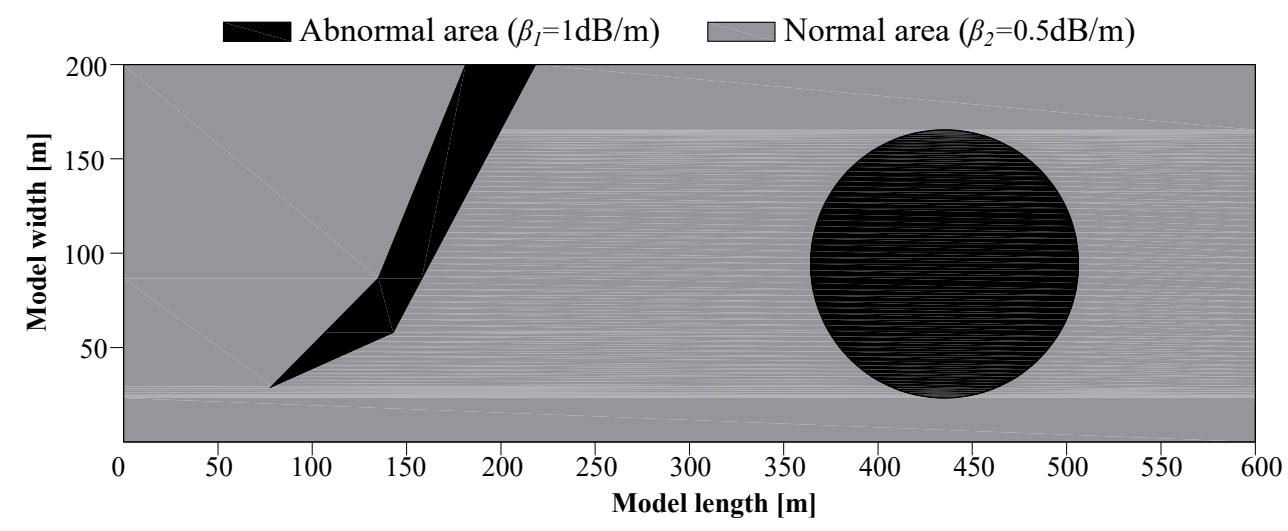

Figure 3. Reconstructed model with anomaly area.

First, the detection area was discretized by meshes, and the layout of observation system was as shown in Figure 4. Among them, the length of each grid was $10 \times 10 \mathrm{~m}$. The distance between signal sources was $30 \mathrm{~m}$ and between acquisition stations was $10 \mathrm{~m}$. The ray coverage angle of each signal source was $20^{\circ}$, the total number of rays was $A=420$, and the total number of grids was $B=1200$. Therefore, the solved model is highly sparse in this observation system.

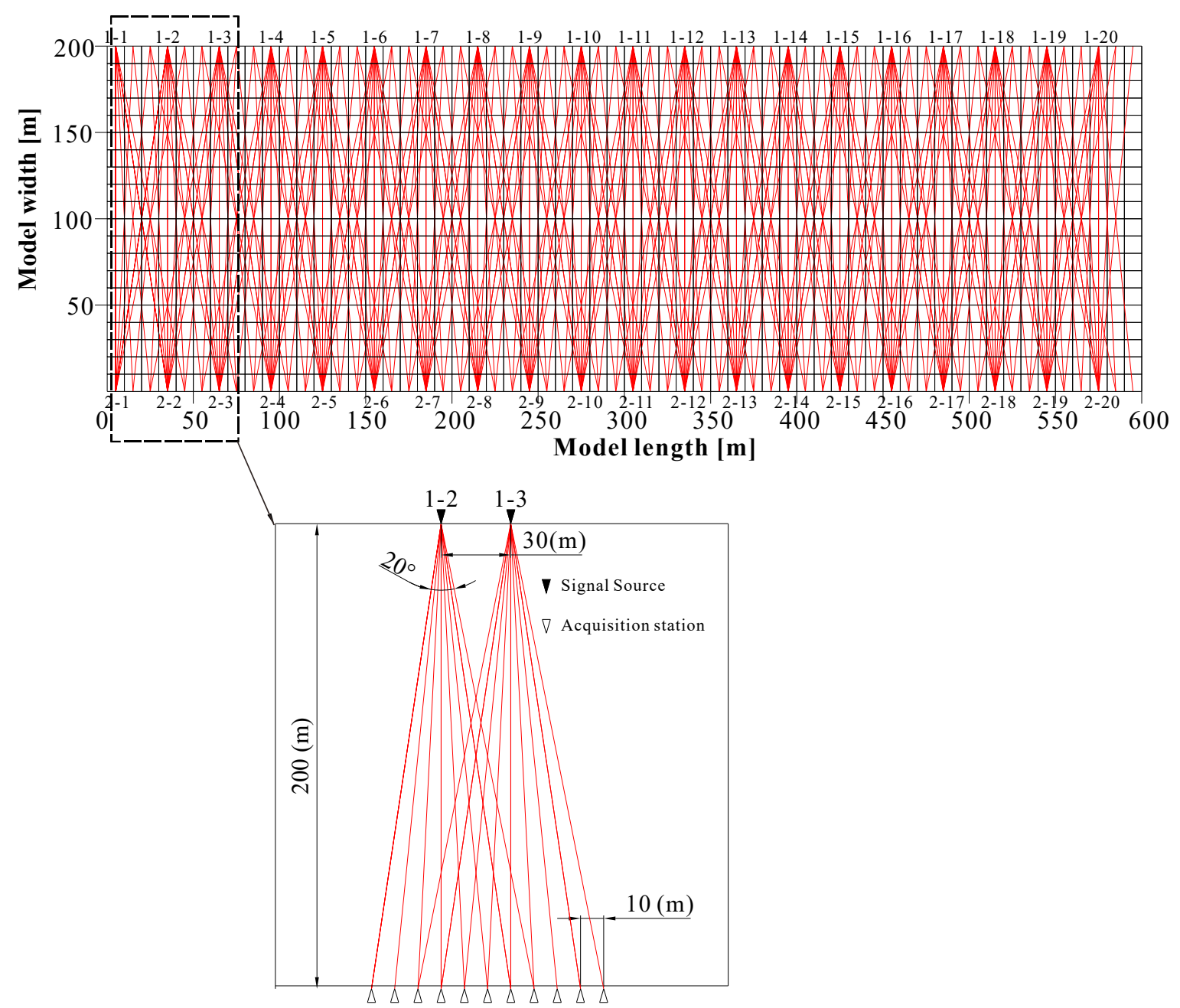

Figure 4. Signal source and acquisition station arrangement in observing systems. 
To investigate the effectiveness of the proposed algorithm, the results of the image reconstructed based on the SGA and AMPGA (without constraint) are shown, respectively. In the numerical experiment, the attenuation coefficients near the upper and lower edges of the model were used as prior information. After 500 iterations of evolution for the different algorithms, the reconstructed results are shown in Figure 5.
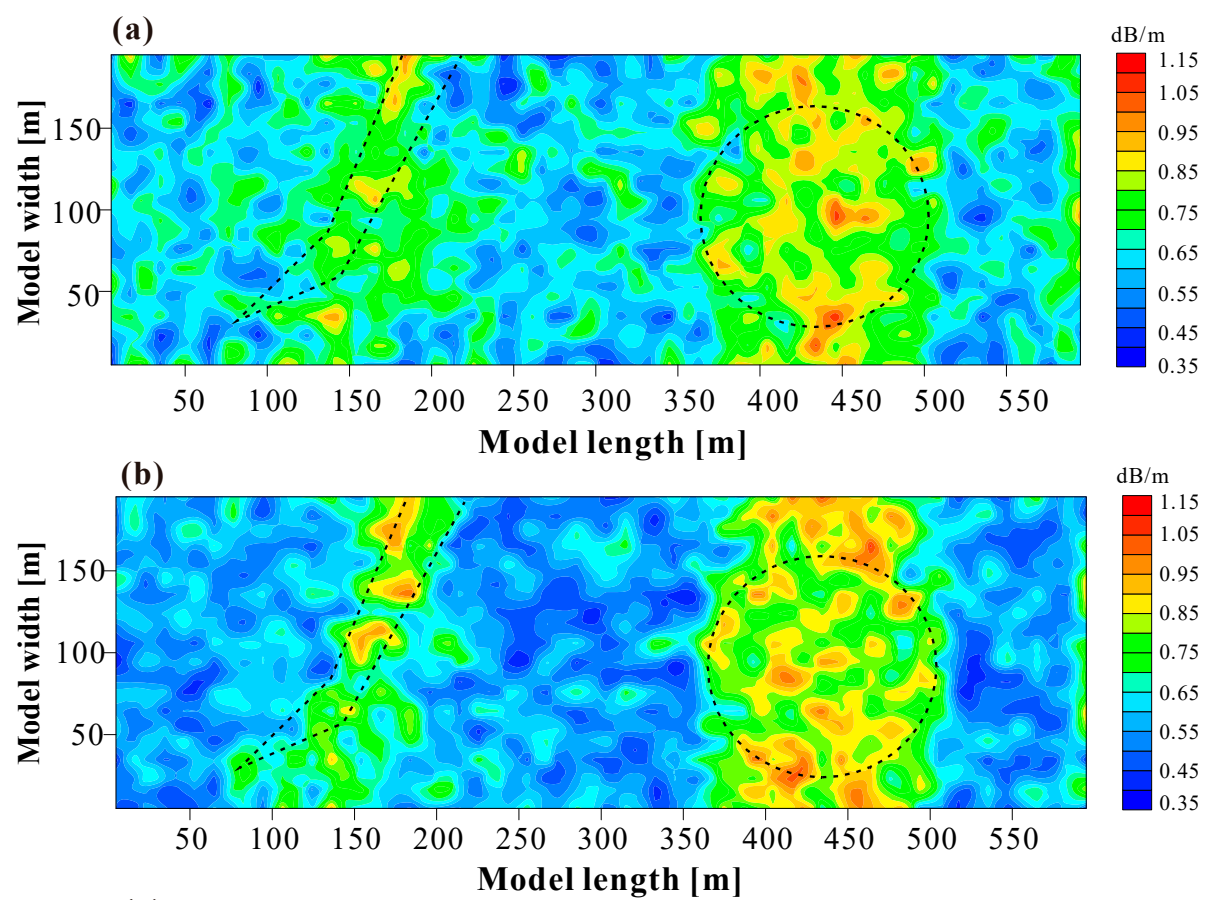

(c)

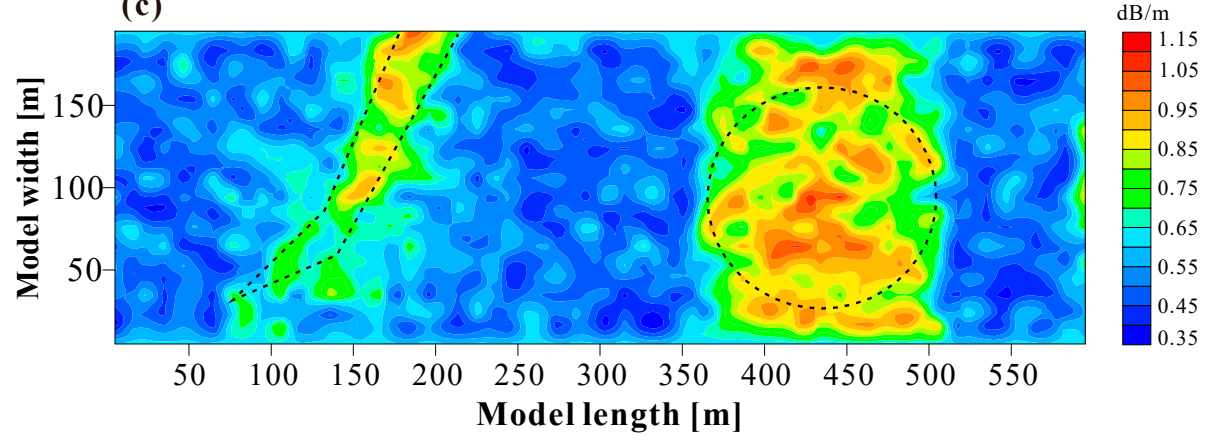

Figure 5. Reconstructed results according to the different algorithms after 500 iterations. (a) Inversion result from the simple genetic algorithm (SGA); (b) inversion result from the AMPGA; (c) inversion result from the AMPGA with constraints.

As indicated in Figure 5, the AMPGA with constraints has a very good performance, and the result is optimally close to the results of the model settings. The inversion image from the SGA has a large area of abnormal structure with a lower resolution, and the boundary is not obvious. The resolution of the reconstructed image has been significantly improved by introducing a variety of adaptive co-evolution strategies, which is shown in Figure $5 \mathrm{~b}$. After introducing a priori information as shown in Figure $5 c$, the quality of AMPGA of the reconstructed image is further improved with a clearer outline, and the distribution of abnormal areas is more concentrated.

To test the stability of these three algorithms, multiple repetitive tests were performed on different algorithms, and the results are shown in Figure 6. Compared with the other two algorithms, curves obtained from the AMPGA with constraints are basically overlapping, so it has the best performance in stability. The comprehensive analysis of the repeated experiments of these three algorithms are shown in Figure 7. After 500 generations of evolution, the average value of RMSE in SGA was reduced 
from 37.01 to $15.89 \mathrm{~dB} / \mathrm{m}$, the average value of RMSE in AMPGA was reduced from 36.15 to $4.44 \mathrm{~dB} / \mathrm{m}$, and the average value of RMSE in AMPGA with constraints was reduced from 36.15 to $3.77 \mathrm{~dB} / \mathrm{m}$. Therefore, compared with SGA, AMPGA can effectively improve the accuracy of tomographic inversion and achieve better imaging results by adding prior information.
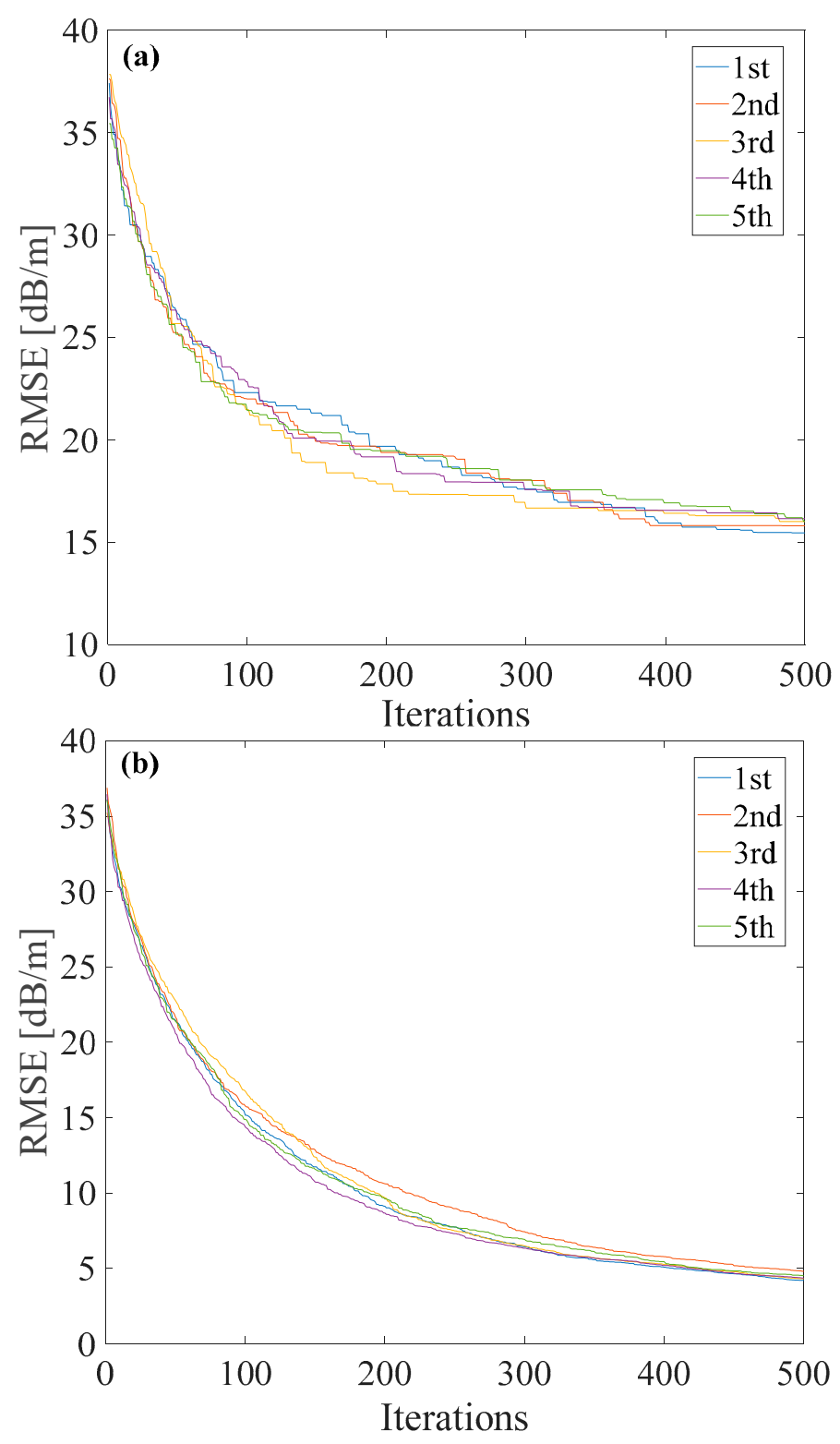

Figure 6. Cont. 


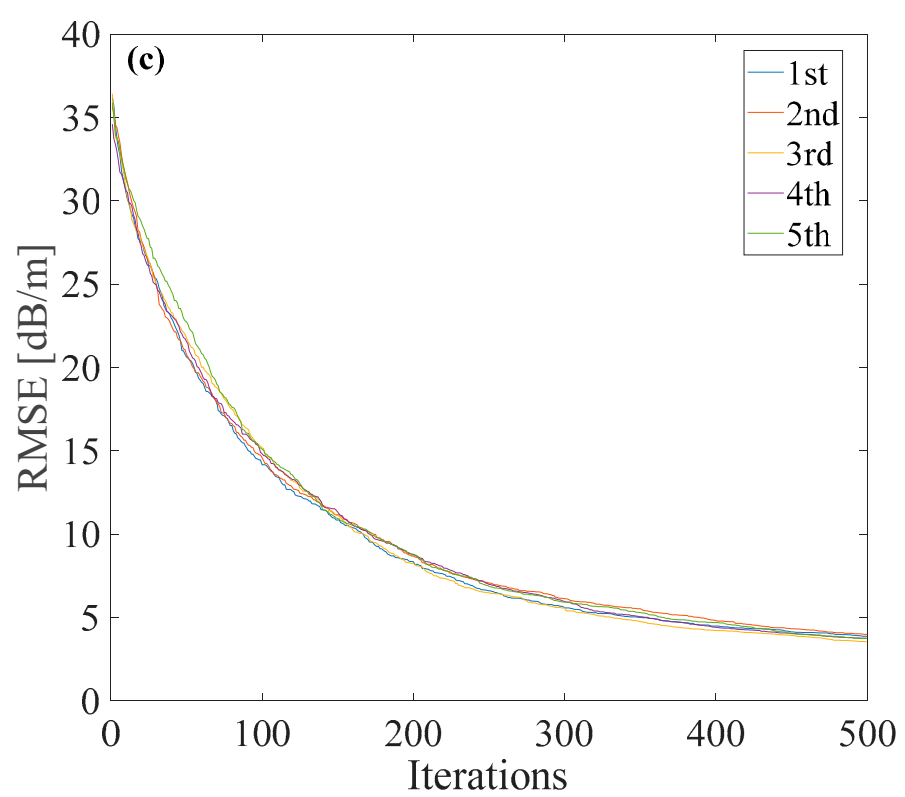

Figure 6. Root mean square error (RMSE) between the computed and the picked attenuation coefficient for the three algorithms after 500 generations of evolution. (a) RMSE from the SGA; (b) RMSE from the AMPGA; (c) RMSE from the AMPGA with constraints.

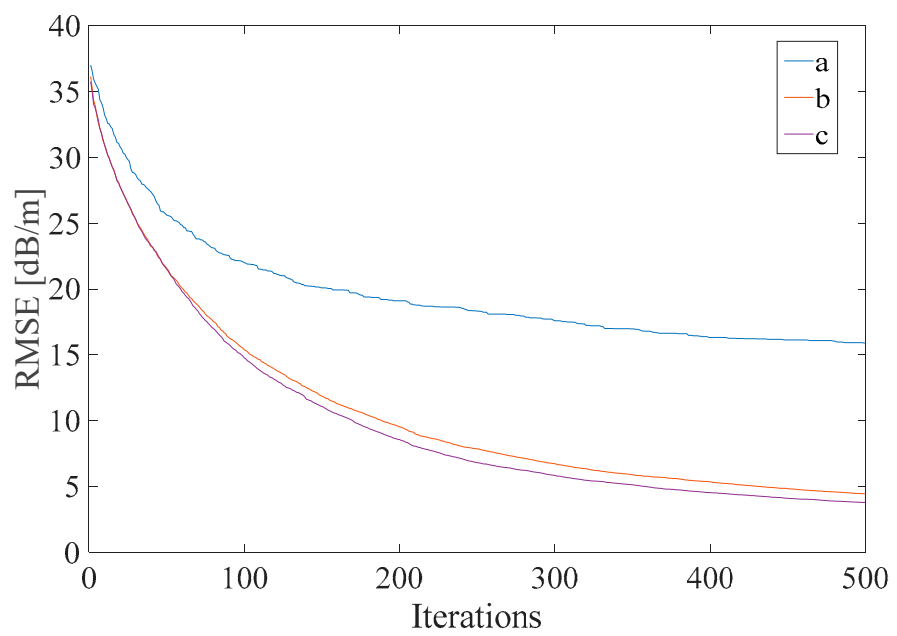

Figure 7. Average of RMSE for these three algorithms according to the multiple tests; curve a is the average RMSE of the SGA; curve $\mathrm{b}$ is the average RMSE of the AMPGA; curve $\mathrm{c}$ is the average RMSE of the AMPGA with constraints.

\section{Field Test}

\subsection{Survey Area}

To verify the significance of the proposed algorithm for coal mining, an electromagnetic wave tomography survey was carried at the Yanzishan Coal Mine, Datong, Shanxi Province, China. The survey area is shown in Figure 8; the inclined length and strike length of the working face in the detection area are 150 and $1000 \mathrm{~m}$, respectively. There are seven faults, as shown in Table 1, and two collapse columns exposed in the detection area. Collapse column X1 is between 250 and $300 \mathrm{~m}$, and collapse column X2 is between 870 and $920 \mathrm{~m}$ in the belt entry. 


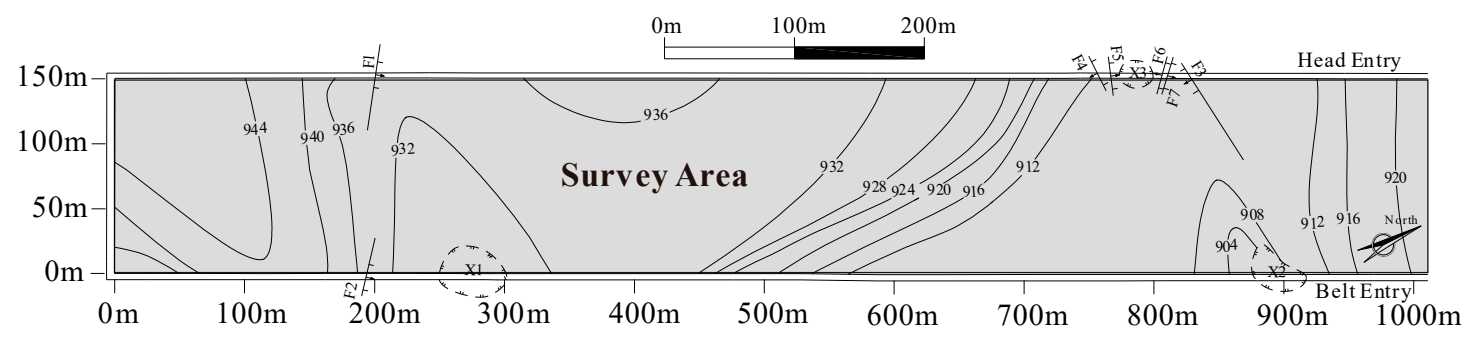

Figure 8. Geological map of the survey area.

Table 1. Faults exposed in the detection area.

\begin{tabular}{cccc}
\hline Faults & Dip $\left(^{\circ}\right)$ & Nature & Drop (m) \\
\hline F1 & 68 & Normal & 4.0 \\
F2 & 36 & Normal & 0.5 \\
F3 & 52 & Normal & 8.0 \\
F4 & 50 & Reverse & 1.0 \\
F5 & 80 & Normal & 1.5 \\
F6 & 60 & Normal & 1.2 \\
F7 & 70 & Normal & 1.4 \\
\hline
\end{tabular}

\subsection{Field Observations}

The instrument used in this exploration is the WKT-E CT system radio mine locator, which is produced by the Chongqing Coal Research Institute in China, and the working frequency is $0.5 \mathrm{MHz}$. The layout of the observation system is shown in Figure 9, and the distance between signal sources was $50 \mathrm{~m}$ and between acquisition stations was $10 \mathrm{~m}$. The ray coverage angle of each signal source was $37^{\circ}$, and the total number of rays was $A=418$.

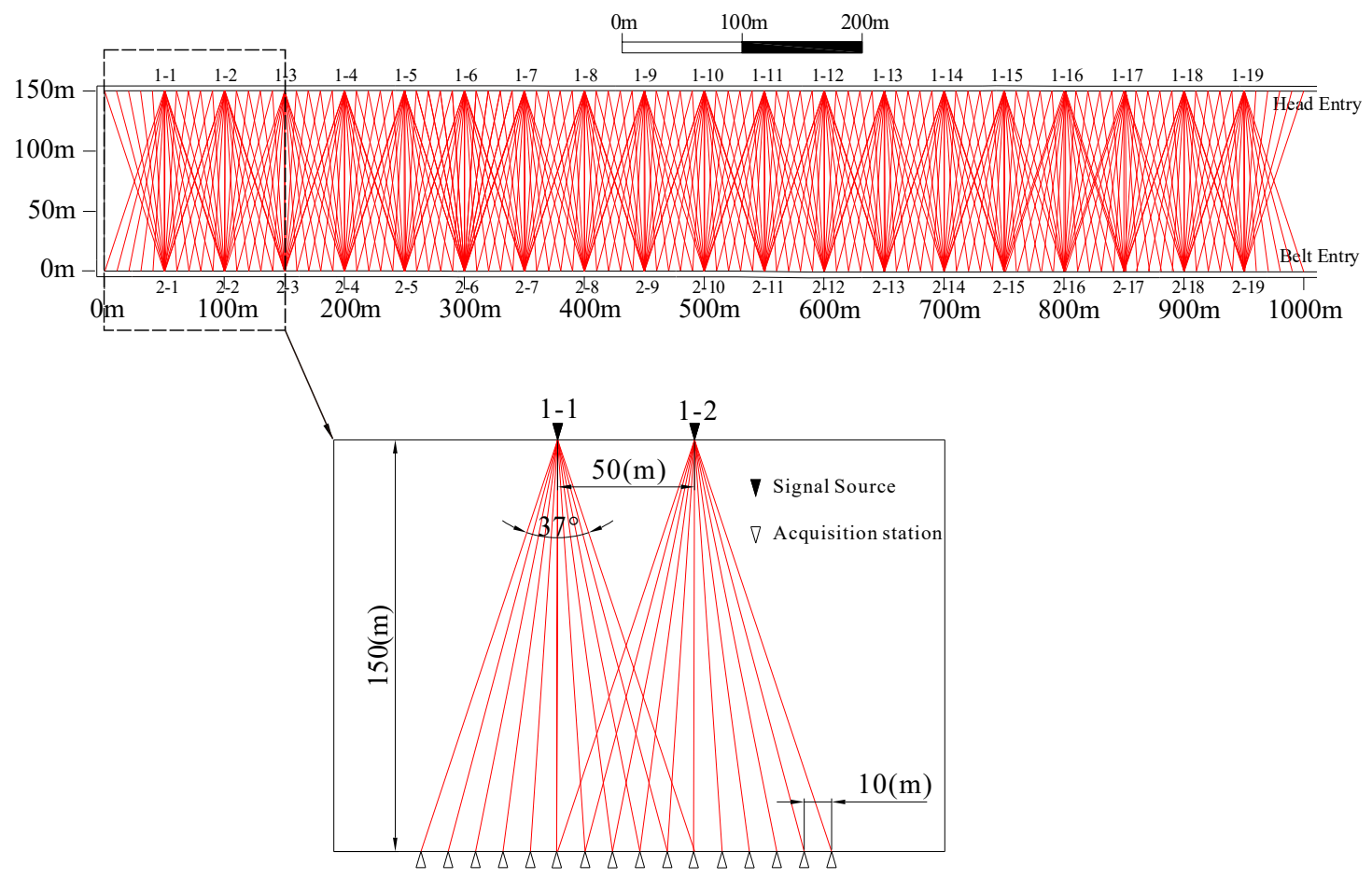

Figure 9. Layout of the observing systems. 


\subsection{Results and Discussion}

After the electromagnetic field intensity data were collected from the survey experiment, the three algorithms were employed to solve the highly sparse model, which can verify the superiority of the proposed algorithm by mutual comparison. First, the detection area was discretized by meshes, and the length of each grid was $10.3 \times 10.3 \mathrm{~m}$. The total number of grids was $B=1470$. The area where the fault is not revealed in the roadway was set as the constraint for the AMPGA, and the value of the absorption coefficient was equal to the average of the entire area.

After 500 iterations of evolution for the three algorithms, the reconstructed results of the detection area are as shown in Figure 10. The results showed that the proposed AMPGA with constraints has the best quality of the image reconstructed with the clearest outline. From Figure 10c, we can see that there are two large areas with a high absorption coefficient, from 170 to $320 \mathrm{~m}$ and 750 to $900 \mathrm{~m}$, which show good correspondence with the exposed structure. Therefore, the exposed structure extends into the working face and penetrates the entire working face, which has a serious influence on mining. For the area of 310-750 m, the inversion result from the AMPGA with constraints shows that most of the area's absorption coefficient is low, which has basically no impact on the mining. However, the inversion result from the SGA has many scattered abnormal areas with a high absorption coefficient, which is hard to judge.

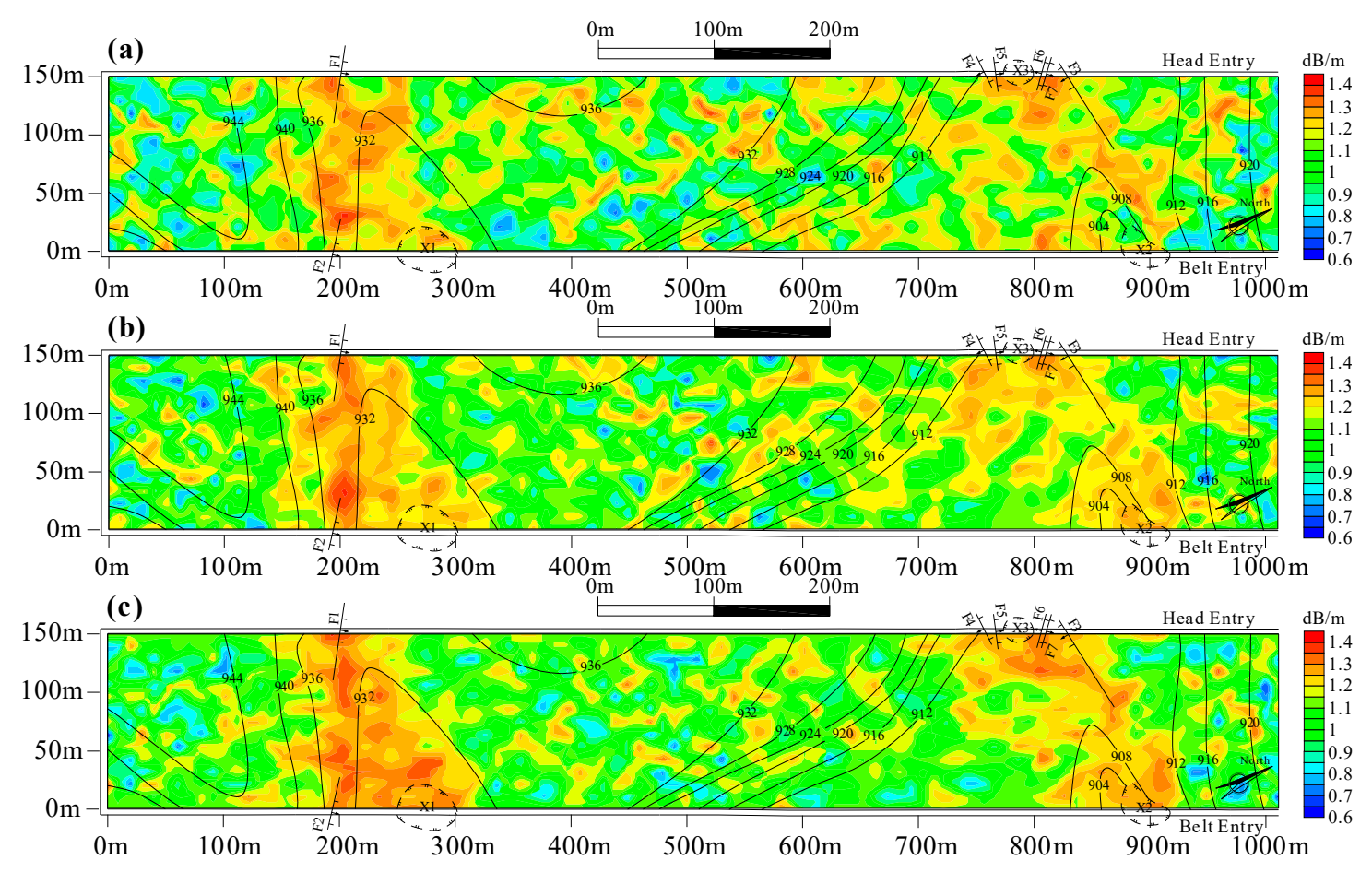

Figure 10. Reconstructed results according to the three algorithms after 500 iterations. (a) Inversion result from the SGA; (b) inversion result from the AMPGA; (c) inversion result from the AMPGA with constraints.

At present, the survey area has been mined from left to right, mining has stopped at the position of $169 \mathrm{~m}$ due to the structure, and the open-off cut has been rebuilt at the position of $326 \mathrm{~m}$. Finally, the coal thickness is too thin to continue mining because of the structure, and the mining has stopped at the position of $760 \mathrm{~m}$. Therefore, the inversion result from the AMPGA with constraints is consistent with the real results.

On the other hand, in order to test the stability of these three algorithms, multiple repetitive tests on different algorithms were performed, as shown in Figure 11. The curves obtained from the AMPGA with constraints are basically overlapping, which has the best stability. A comprehensive 
analysis of the repeated experiments of these three algorithms is available, as shown in Figure 12. The average RMSE of the SGA was reduced from 16.30 to $6.90 \mathrm{~dB} / \mathrm{m}$, the RMSE of the AMPGA was reduced from 15.51 to $2.94 \mathrm{~dB} / \mathrm{m}$, and the RMSE of the AMPGA with constraints was reduced from 15.97 to $2.57 \mathrm{~dB} / \mathrm{m}$. Therefore, the AMPGA with constraints has a better stability and accuracy than the other two algorithms.

Furthermore, the SGA begins to converge, and the error remains basically stable after 200 generations of evolution. While the error of the AMPGA with constraints continues to decrease after 200 generations of evolution, it will not converge until after 450 generations. Therefore, the AMPGA with constraints has a better convergence effect than SGA.
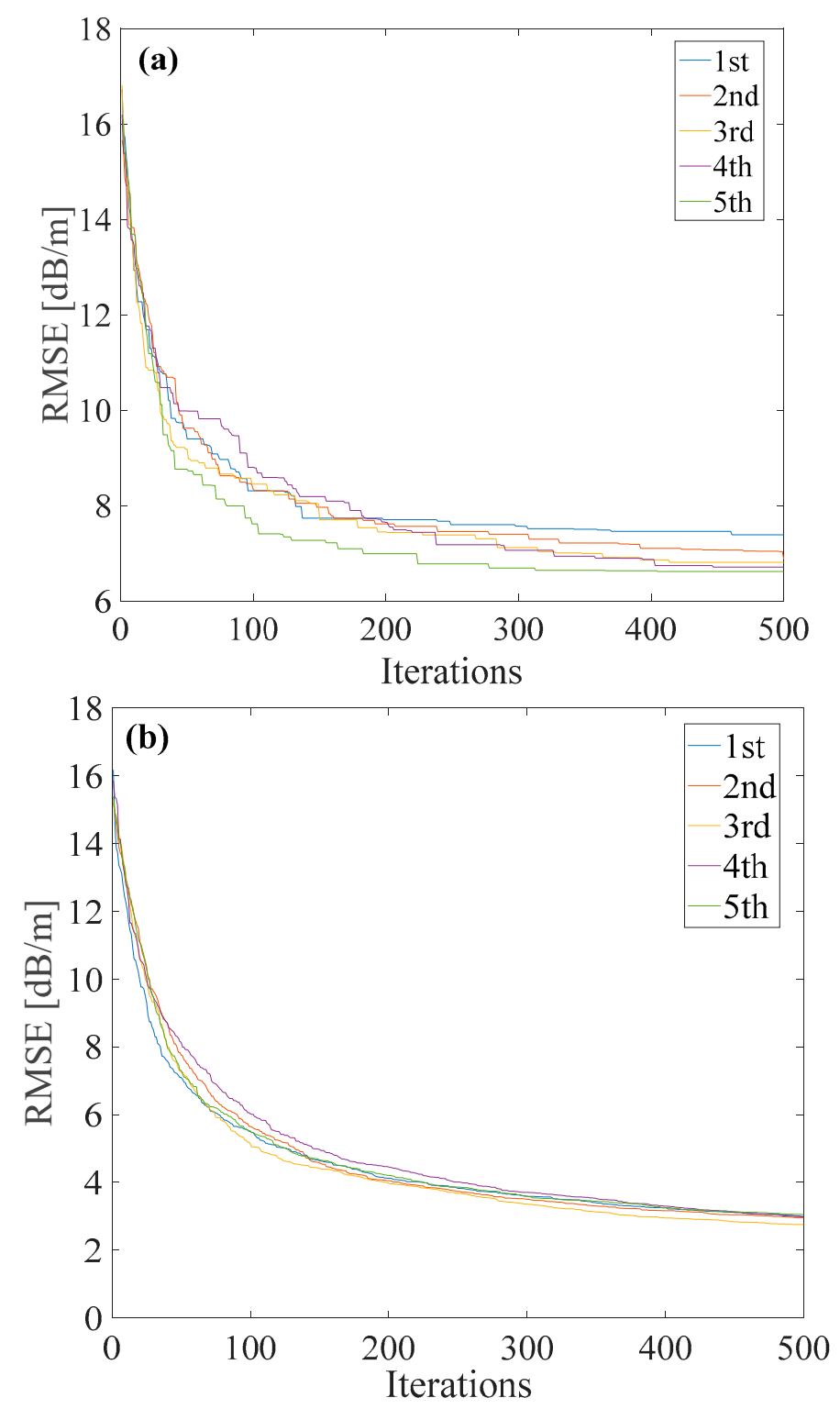

Figure 11. Cont. 


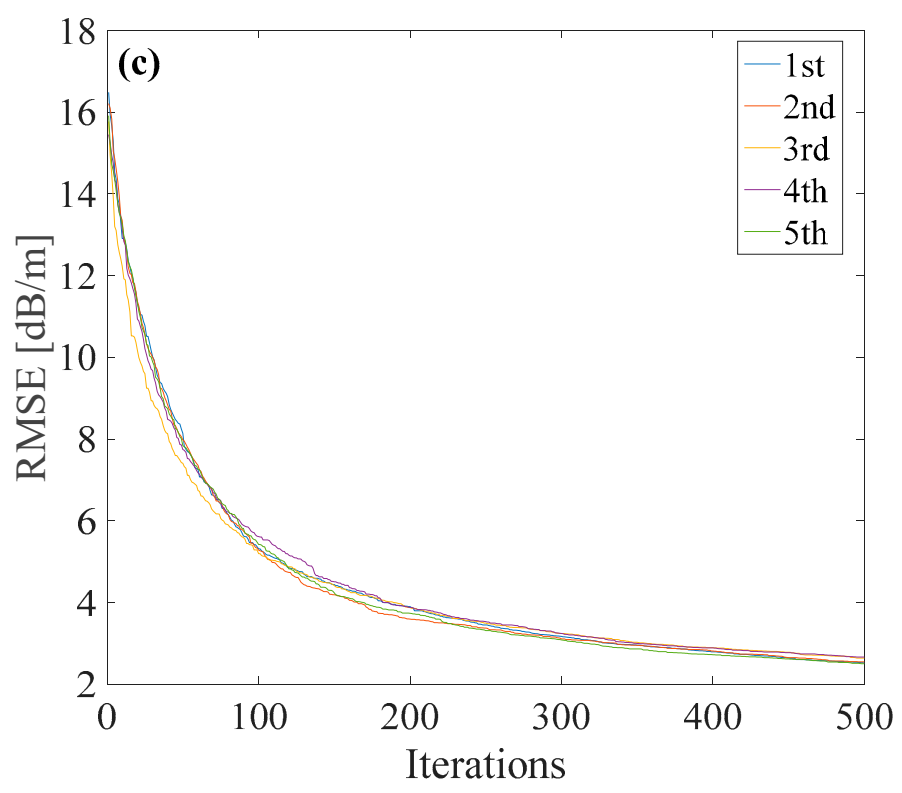

Figure 11. The RMSE between the computed and the picked attenuation coefficient for the three algorithms after 500 generations of evolution. (a) RMSE from the SGA; (b) RMSE from the AMPGA; (c) RMSE from the AMPGA with constraints.

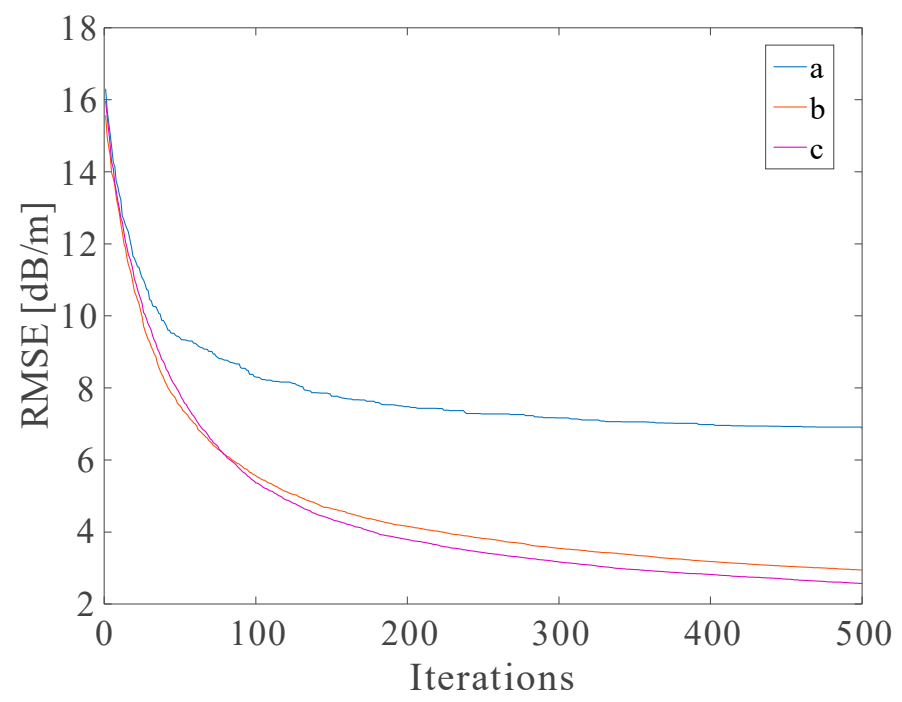

Figure 12. The average RMSE for these three algorithms according to the multiple tests; curve a is the average RMSE of the SGA; curve $\mathrm{b}$ is the average RMSE of the AMPGA; curve $\mathrm{c}$ is the average RMSE of the AMPGA with constraints.

\section{Conclusions}

Due to incomplete projection data, searching for the accurate and stable global optimal solution for the objective function has a significant impact on the effect of image reconstruction. In this research, the proposed algorithm fully combines the strategies of multi-group adaptive co-evolution and the constraints of prior information, which effectively improves the global and local search ability of the SGA. The results of the numerical experiment and field tests show that the proposed algorithm has better performance on tomography image reconstruction than SGA and AMPGA, and the reconstruction accuracy and stability are higher than those of the other two.

The proposed AMPGA with constraints has the ability to solve the premature convergence problem of SGA and significantly reduce the error of the inversion model. Therefore, this study 
provides an important basis for the mining plans, and it also presents a new strategy to obtain a more accurate and stable inversion result in underground resource exploration.

Author Contributions: Conceptualization, C.G., Z.Y.; Data curation, C.G., X.W., and T.T.; Formal analysis, C.G.; X.W.; Investigation, C.G., Z.Y., and T.T.; Methodology, C.G., Z.Y.; Writing-original draft, C.G., K.Z.; Writing-review \& editing, C.G.; Z.Y., and K.Z.

Funding: This work was supported by the Fundamental Research Funds for the Central Universities [No. 2017CXNL01].

Conflicts of Interest: The authors declare no conflict of interest.

\section{References}

1. Liu, R.; Chen, S.; Ji, G.; Zhao, B.; Li, Q.; Su, M. Interactive stratigraphic structure visualization for seismic data. J. Vis. Lang. Comput. 2018, 48, 81-90. [CrossRef]

2. Liu, $\mathrm{X}$.; Liu, Z. A novel algorithm based on $\mathrm{L}_{1}-\mathrm{L}_{\mathrm{p}}$ norm for inverse problem of electromagnetic tomography. Flow Meas. Instrum. 2019, 65, 318-326. [CrossRef]

3. Poltschak, S.; Freilinger, M.; Feger, R.; Stelzer, A.; Hamidipour, A.; Henriksson, T.; Semenov, S. A multiport vector network analyzer with high-precision and realtime capabilities for brain imaging and stroke detection. Int. J. Microw. Wirel. Technol. 2018, 10, 605-612. [CrossRef]

4. Greenfield, R.J.; Wu, T. Electromagnetic wave propagation in disrupted coal seams. Geophysics 1991, 56, 1571-1577. [CrossRef]

5. Hatherly, P. Overview on the application of geophysics in coal mining. Int. J. Coal Geol. 2013, 114, 74-84. [CrossRef]

6. Katterbauer, K.; Hoteit, I.; Sun, S. EMSE: Synergizing EM and seismic data attributes for enhanced forecasts of reservoirs. J. Petrol. Sci. Eng. 2014, 122, 396-410. [CrossRef]

7. Katterbauer, K.; Arango, S.; Sun, S.; Hoteit, I. Enhanced characterization of reservoir hydrocarbon components using electromagnetic data attributes. J. Petrol. Sci. Eng. 2016, 140, 1-15. [CrossRef]

8. Yang, Z.; Wu, X.; Yao, W.L.; Guo, C.F. Research on CT inversion adopting prior constraints and singularity model for a longwall panel. J. China Min. Technol. 2017, 46, 988-996. [CrossRef]

9. Holland, J. Adaptation in Natural and Artificial Systems; University of Michigan Press: Ann Arbor, MI, USA, 1975.

10. Lv, R.; Xiao, L.; Jiang, X.; Feng, M.; Yang, F.; Tian, J. Optimization of Red Luminescent Intensity in Eu3+-Doped Lanthanide Phosphors Using Genetic Algorithm. ACS Biomater. Sci. Eng. 2018, 4, 4378-4384. [CrossRef]

11. Mariane, R.; Eduardo, F.; Fernando, R.; Henrique, A.; Helio, B. Optimization of Mass Concrete Construction Using a Twofold Parallel Genetic Algorithm. Appl. Sci. 2018, 8, 399. [CrossRef]

12. Rynkevic, R.; Ferreira, J.; Martins, P.; Parente, M.; Fernandes, A. Linking hyperelastic theoretical models and experimental data of vaginal tissue through histological data. J. Biomech. 2019, 82, 271-279. [CrossRef] [PubMed]

13. Kumar, A.; Guria, C.; Pathak, A.K. Optimal cultivation towards enhanced algae-biomass and lipid production using Dunaliella tertiolecta for biofuel application and potential $\mathrm{CO}_{2}$ bio-fixation: Effect of nitrogen deficient fertilizer, light intensity, salinity and carbon supply strategy. Energy 2018, 148, 1069-1086. [CrossRef]

14. Yamada, M. 1/f Noise in the Simple Genetic Algorithm Applied to a Traveling Salesman Problem. Fluct. Noise Lett. 2017, 16, 1750026. [CrossRef]

15. Sadi, M.; Shahrabadi, A. Evolving robust intelligent model based on group method of data handling technique optimized by genetic algorithm to predict asphaltene precipitation. J. Petrol. Sci. Eng. 2018, 171, 1211-1222. [CrossRef]

16. Guo, C.F.; Yang, Z.; Chang, S.; Ren, T.; Yao, W. Precise Identification of Coal Thickness by Channel Wave Based on a Hybrid Algorithm. Appl. Sci. 2019, 9, 1493. [CrossRef]

17. Soupios, P.; Akca, I.; Mpogiatzis, P.; Basokur, A.T.; Papazachos, C. Applications of hybrid genetic algorithms in seismic tomography. J. Appl. Geophys. 2011, 75, 479-489. [CrossRef]

18. He, F.; Zhang, X.; Chen, B.; Fok, M. Inversion of the Earth's plasmaspheric density distribution from EUV images with genetic algorithm. Chin. J. Geophys. 2012, 55, 29-35. [CrossRef]

19. Moro, G.; Pipan, M.; Gabrielli, P. Rayleigh wave dispersion curve inversion via genetic algorithms and Marginal Posterior Probability Density estimation. J. Appl. Geophys. 2007, 61, 39-55. [CrossRef] 
20. Wan, L.; Lin, T.; Lin, J.; Jiang, C.; Ji, Y. Joint inversion of MRS and TEM data based on adaptive genetic algorithm. Chin. J. Geophys. 2013, 56, 3728-3740. [CrossRef]

21. Hao, Y.; Yang, D.; Cheng, Y. Reservoir parameter inversion of $\mathrm{CO}_{2}$ geological sequestration based on the self-adaptive hybrid genetic algorithm. Chin. J. Geophys. 2016, 59, 4234-4245. [CrossRef]

22. Ranjan, A.; Misra, P.; Dwivedi, B.; Sahu, H. Studies on Propagation Characteristics of Radio Waves for Wireless Networks in Underground Coal Mines. Wirel. Pers. Commun. 2017, 97, 2819-2832. [CrossRef]

23. Zhang, H.; Li, Z.; Zou, H. Application of radio wave transmission in coal mine. Coal Sci. Technol. 2002, 30, 28-30. [CrossRef]

24. Zhang, Y.; Han, G.; Zhang, W.; Sheng, J. Electromagnetic Mode Theory of Periodically-Loaded Oversized Imperfect Waveguide and Its Application to the Propagation of Radio Waves in Long Wall Coal Mining Face Tunnels. IEEE. Trans. Antenn. Propag. 2010, 58, 1816-1822. [CrossRef]

25. Du, H.; Chen, W.; Zhu, Q.; Liu, S.; Zhou, J. Identification of weak peaks in X-ray fluorescence spectrum analysis based on the hybrid algorithm combining genetic and Levenberg Marquardt algorithm. Appl. Radiat. Isotopes. 2018, 141, 149-155. [CrossRef] [PubMed]

26. Fu, Y.; Wang, H.; Huang, M.; Ding, J.; Tian, G. Multiobjective flow shop deteriorating scheduling problem via an adaptive multipopulation genetic algorithm. Proc. Inst. Mech. Eng. Part B J. Eng. Manuf. 2018, 232, 2641-2650. [CrossRef]

27. Li, Y.; Zeng, X.; Han, L.; Wang, P. Two coding based adaptive parallel co-genetic algorithm with double agents structure. Eng. Appl. Artif. Intell. 2010, 23, 526-542. [CrossRef]

28. Zandieh, M.; Karimi, N. An adaptive multi-population genetic algorithm to solve the multi-objective group scheduling problem in hybrid flexible flowshop with sequence-dependent setup times. J. Intell. Manuf. 2011, 22, 979-989. [CrossRef]

29. Ben Mansour, I.; Basseur, M.; Saubion, F. A multi-population algorithm for multi-objective knapsack problem. Appl. Soft Comput. 2018, 70, 814-825. [CrossRef]

30. Huang, Y.; Ma, X.; Su, S.; Tang, T. Optimization of Train Operation in Multiple Interstations with Multi-Population Genetic Algorithm. Energies 2015, 8, 14311-14329. [CrossRef]

31. Ye, Y.; Gao, M.; Ma, Y.; Shao, W.; Chen, W.; Yan, Y.; Ren, H. Multi-population genetic algorithm for peak-to-average power ratio suppression in an optical OFDM transmission system. Appl. Opt. 2018, 57, 10191-10197. [CrossRef]

32. Mokshin, A.; Mokshin, V.; Sharnin, L.M. Adaptive genetic algorithms used to analyze behavior of complex system. Commun. Nonlinear Sci. 2019, 71, 174-186. [CrossRef]

33. Shojaedini, E.; Majd, M.; Safabakhsh, R. Novel adaptive genetic algorithm sample consensus. Appl. Soft Comput. 2019, 77, 635-642. [CrossRef]

(C) 2019 by the authors. Licensee MDPI, Basel, Switzerland. This article is an open access article distributed under the terms and conditions of the Creative Commons Attribution (CC BY) license (http://creativecommons.org/licenses/by/4.0/). 